\title{
Joint Sounds Analysis and its Relationship with the Temporomandibular Disorders Severity
}

\author{
Carolina A. Rodrigues*, Lais V. Magri, Melissa de O. Melchior, Takami H. Hotta, Dr. Marcelo 0. \\ Mazzetto \\ School of Dentistry of Ribeirão Preto, Department of Restorative Dentistry, University of São Paulo, Brazil
}

Received: June 23, 2014; Accepted: August 04, 2014; Published: August 24, 2014

*Corresponding author: Carolina Almeida Rodrigues, School of Dentistry of Ribeirão Preto, Department of Restorative Dentistry, University of São Paulo, Brazil, CEP:14040-904, Phone: +55-16-3602-4087 ; Fax: +55-16-3602-478; E-mail: carolinaalmeidarodrigues@hotmail.com

\begin{abstract}
Objectives: Analyze parameters which enable the evaluation of the severity of joint sounds during Electrovibratography (EVG): Amplitude Peak (AP) and Frequency Peak (FP); analyze the correlation between the data of EVG with Temporomandibular Disorder (TMD) severity given by Craniomandibular Index (CMI).

Methods: 79 subjects with TMD were submitted to clinical examinations of the Research Diagnostic Criteria (RDC/TDM axis I) for diagnosis of TMD, CMI to investigate the severity of the dysfunction. 18 patients were part of the control group and all patients underwent electrovibratography.

Results: The AP was greater in left Temporomandibular Joint (TMJ) (3.71 \pm 7.73 opening and $2.71 \pm 3.49$ closing) as well as the opening movement ( $3.71 \pm 7.73$ left TMJ and 2:42 \pm 5.19 right TMJ). From the FP, higher values were also found in the left joint $(71.31$ \pm 70.41 opening and $47.99 \pm 52.46$ closing) and during the opening movement ( $71.31 \pm 70.41$ left TMJ and $52.09 \pm 45.35$ right TMJ). For the control group there were no significant differences. Furthermore correlation between the CMI and the FP in the left TMJ during opening was found $(p=0.02)$.
\end{abstract}

Conclusion: Joints of subjects with TMD had a higher AP and FP as compared to a control group and the left TMJ showed higher values. The frequency peak may be related to the severity of the disorder, and the EVG assists in the formulation of diagnosis and assessment of severity of articular TMD.

Keywords: Temporomandibular joint dysfunction syndrome; Temporomandibular joint; Sounds; Severity

\section{Introduction}

Temporomandibular Disorders (TMD) are changes in the temporomandibular joints, masticatory muscles, or both, affecting the relative share of the population. Characteristics are frequent musculoskeletal pain expressed in the region of the face, head, neck and ears, articular sounds when opening and/or closing mouth, changes in patterns of jaw movements, such as limitations of these movements or deviations and deflections [1,2].

The pathogenesis of TMD is considered multifactorial [3], and considering this plus a wide variety of signs and symptoms, it is appropriate that the possible causes are analyzed to formulate an accurate diagnosis.

The Temporomandibular Joint (TMJ) often presents many sounds, which is frequently noticed when there are some internal derangements of the joint. The clicks and crepitations are the most common sounds observed. The sound event occurs when the disc is partially or completely displaced, as a result of tissue morphological and functional changes; there may be also typical sounds that are associated with degenerative joint $[4,5]$.

The recording of sounds and vibrations of TMDs may be important for the diagnosis of patients with suspected internal joint disorder. Therefore, the study of specific parameters, such as the repetitive nature of the sounds, the jaw position in the opening and closing of the mouth, at the specific time of happening the noise added to the remaining clinical and instrumental, can provide information for precise treatment.

The electrovibratography is used to study joint sounds which is termed to be a method of accurate analysis, recording the total energy of the noise (Hertz), the amplitude peak (Pascal), frequency peak (Hertz), duration and mandibular position in which they occur, making possible to the researchers to correlate types of vibrations with specific dysfunctions [6]. The aim of the study is to analyze the relationship between the Electrovibratography (EVG) parameters of joint sounds and TMD severity.

\section{Methods}

After the approval by Research Ethics Committee from the School of Dentistry of Ribeirão Preto (University of São Paulo USP, Brazil) under protocol number 0041.0.138.000-11, a group of the 97 subject (196 TMJ) was selected, in which 79 subjects (total of $158 \mathrm{TMJ}$ ) had pain in the TMJ region not associated with orofacial muscle pain during palpation and 18 subjects (total of 36 TMJ) without signs and symptoms of TMD were included in the control group. Patients with dental absences above two teeth were excluded from the study; patients who complain only of muscle disorders; patients with psychiatric disorders; tumors; patients with signs and symptoms of fibromyalgia; and pregnant patients. 
The subjects with TMD underwent clinical examination by a single examiner, according to the criteria of axis I index RDC/ TMD [7] and severity of dysfunction was assessed using the Craniomandibular Index (CMI) [8].

Subsequent to the evaluation of diagnostic protocols and CMI, the study was conducted through the joint sounds electrovibratography (EVG), by a computerized - SonoPAK QSSystem (BioReserch, INC, Milwaukee, Wisconsin).

Records of joint vibration analysis was performed according to protocol are used for Mazzetto MO, Hotta, Mazzetto RG [9]. After accommodation of the patient, the orientation of look is adjusted in the direction of the screen, and the maximum comfortable opening measures including the overbite and the lateral deflection of the mandible during mouth opening are noted, using a digital caliper (Mitutoyo, Brazil); positioning of the amplifier and piezoelectric sensors was performed in the subject (Figure 1). The examination was performed with the patient doing movements of mouth opening and closing following the simulation provided by software, with metronome in real time on the computer screen. After a period of 10 seconds, the test is recorded in the computer. The software by BioResearc is used to find the 'points of noise', through the cursor "Find Vibration" in the software toolbar.

For this study, we analyzed the numerical data of the amplitude peak (point of highest intensity vibration) and frequency peak (the point of highest frequency) of joint sounds. These numerical data were used in the statistical analysis. The opening and closing movements were analyzed separately (Figures 2 and 3).

All data were organized into spreadsheets and inserted into BioEstat 5.0 for statistical analysis. For analysis of amplitude peak and frequency peak during the opening and closing movement of the jaw joint and between left and right test, the data was applied to the analysis of variance with a standard. To check the correlation between severity of TMD and the joint sounds, the "spearman correlation" (nonparametric) test was applied, between the amplitude peak and frequency peak found in each joint with CMI.

After collecting all the data, each patient individually received the necessary treatments.

\section{Results}

A sample of 97 subjects was composed of 80 female subjects $(82.47 \%)$ and 17 males $(17.52 \%)$ with an average age of 30.83 . From the RDC/TMD subjects with TMD were classified as follows: 15 (18.75\%) in group II and none in group III alone (0\%). The other patients with associated diagnoses were classified in more than one group. Thirty five subjects $(43.75 \%)$ were rated in groups I and II, and 12 (15\%) were classified in group I and III, nine $(11.25 \%)$ subjects in groups II and III, and the association of the three diagnostic groups was observed in 8 volunteers (10\%). The TMD severity of these patients assessed using the CMI, can have values from 0 to 1 . The CMI of the sample had a mean $(0.519$ $\pm 0.175)$.

In the present study, according to EVG, 72 left TMJ noise presented in the opening, 71 right TMJ noise presented in the opening, 71 left TMJ noise presented in the closing jaw movement and 70 right TMJ noise presented in the closing, totalizing 143 joints with noise in opening and 141 joints with noise in closing. Furthermore, eletrovibratography was used to evaluate the point of highest intensity of vibration of joint sounds, equivalent to peak amplitude, measured in Pascals (Pa). The mean value showed in the opening point of higher intensity on left TMJ (Table 1 and Figure 4). The closing movement of the left joint also showed higher peak amplitude in relation to right TMJ (Table 1 and Figure 5). In addition, there were no differences when comparing

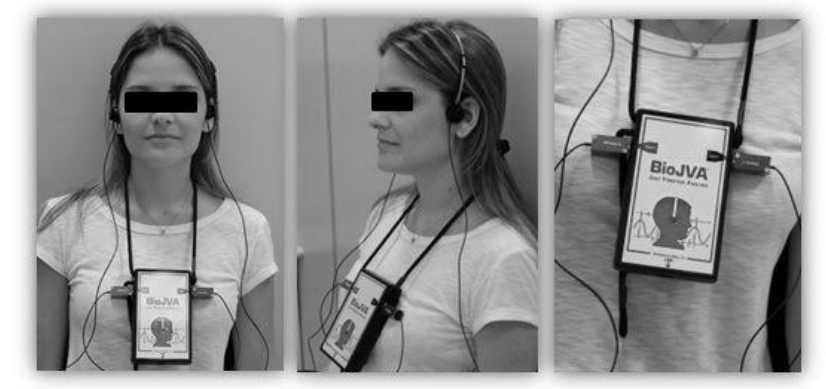

Figure 1: Placement of piezoelectric sensors on the joints and amplifier with cables connected.

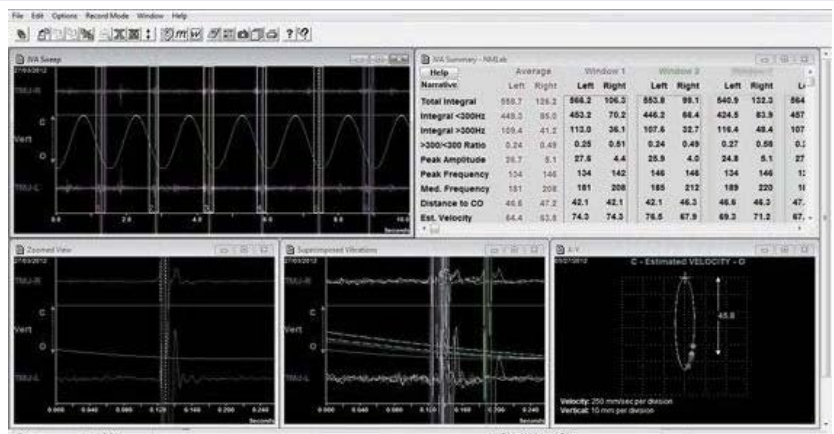

Figure 2: SonoPAK program, the upper graph indicates the vibrations of higher intensities in the peak amplitude (JVA sweep window, featured 1-6), and the lower indicates, the times when the sounds occur during mandibular opening. The dots appearing between the start of the opening and final closing, represent the noise resulting from contact between the teeth.

\begin{tabular}{|c|c|c|c|c|c|c|c|c|c|c|c|c|c|c|}
\hline \multicolumn{15}{|c|}{ 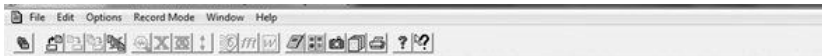 } \\
\hline Help & \multicolumn{2}{|c|}{ Average } & \multicolumn{2}{|c|}{ Window 1} & \multicolumn{2}{|c|}{ Wneow 2} & \multicolumn{2}{|c|}{ masw 2} & \multicolumn{2}{|c|}{ Window 4} & \multicolumn{2}{|c|}{ Wincows 5} & \multicolumn{2}{|c|}{ Window 6} \\
\hline Narrative & Lett & & Lot & & Left & Right & Lot & Right & Left & Right & Left & Right & Lett & Right \\
\hline Total Integral & 36.5 & 9.9 & 63.8 & 14.6 & 93.7 & 9.9 & 21.7 & 8.0 & 21.5 & 6.5 & 68.3 & 11.7 & 30.2 & 9.0 \\
\hline Integral $<300 \mathrm{~Hz}$ & 35.6 & 9.5 & 52.6 & 14.0 & 32.8 & 9.5 & 21.1 & 7.4 & 20.8 & 5.9 & 57.0 & 11.1 & 29.5 & \\
\hline tegral >300Hz & 0.9 & 0.7 & 1.3 & 0.6 & 0.9 & 0.5 & 0.6 & 0.6 & 0.6 & 0.6 & 1.3 & 0.6 & 0.7 & \\
\hline$>3000<300$ Ratio & 0.03 & 0.07 & 0.02 & 0.04 & 0.03 & 0.05 & 0.03 & 0.08 & 0.03 & 0.10 & 0.02 & 0.06 & 0.02 & 0.14 \\
\hline $\mathrm{ak}$ A $\mathrm{C}$ & 4.6 & 1.0 & 6.5 & 1.4 & 4.3 & 1.1 & 2.8 & 0.9 & 2.9 & 0.7 & 7.2 & 1.1 & 4.2 & \\
\hline Peak Frequency & 83 & 64 & 87 & 68 & n & 64 & 80 & 72 & 72 & 68 & 87 & 25 & 80 & \\
\hline Med. Frequency & 95 & 80 & 99 & 80 & 91 & 76 & 87 & 76 & 83 & 72 & 99 & 83 & 91 & \\
\hline Distance to $\mathrm{co}$ & 3.5 & & 4.2 & 4.4 & 0.7 & 0.8 & 3.0 & & 1.2 & & 2.4 & 2.8 & 9.5 & \\
\hline Est Velocity & & 35.1 & 47.9 & 48.3 & 19.9 & 19.0 & 39.6 & & 23.0 & & 36.4 & 38.2 & 68.5 & \\
\hline $\begin{array}{l}\text { Max. Opening } \\
\text { Lat. Deflection }\end{array}$ & & t. & & & & & & & & & & & & \\
\hline $\begin{array}{l}\text { notialfection } \\
\text { titial Exam }\end{array}$ & & & & & & & & & & & & & & \\
\hline
\end{tabular}

Figure 3: Program SonoPAK with the numerical data obtained by marking joint sounds through the JVA Summary. The values used in this study were the amplitude peak, measured in pascals, and frequency peak, in hertz. 


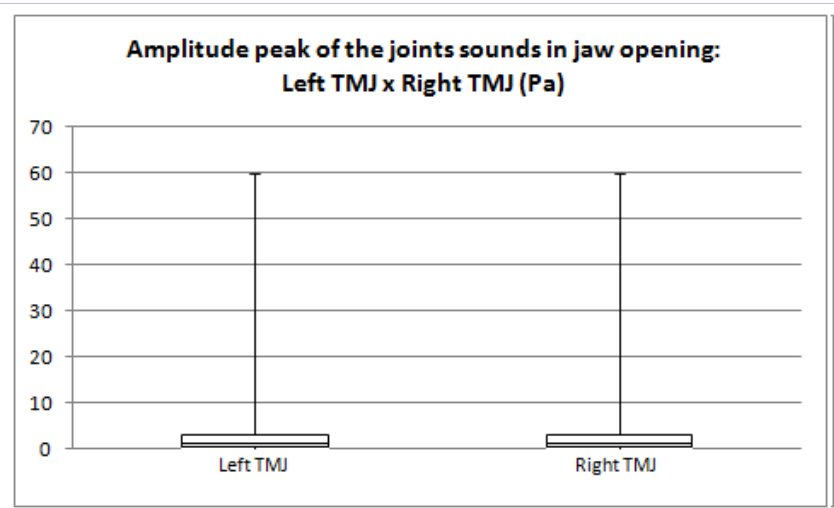

Figure 4: Mean and standard deviation of the amplitude peak of the joint sounds during jaw opening, TMJ left and right TMJ.

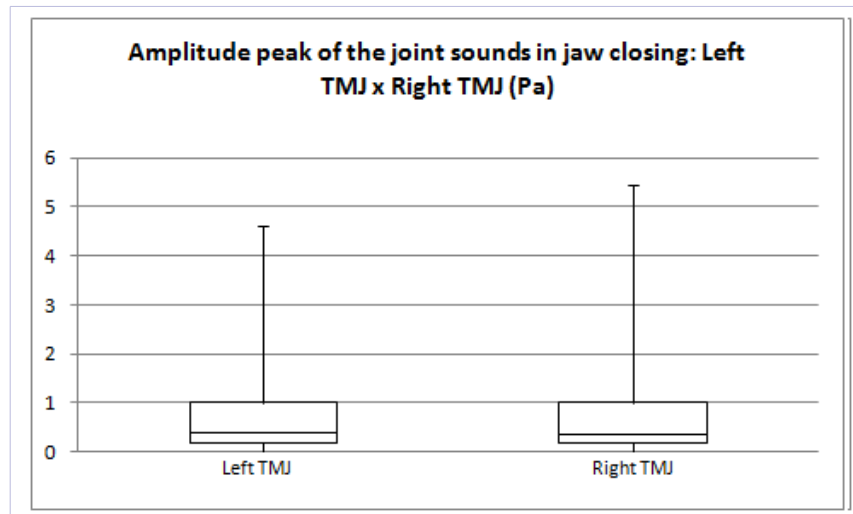

Figure 5: Mean and standard deviation of the amplitude peak of the joint sounds during jaw closing, TMJ left and right TMJ.

Table 1: Amplitude peak of joint sounds in subjects with temporomandibular disorders.

\begin{tabular}{|l|c|c|c|c|c|c|c|c|}
\hline & $\begin{array}{c}\text { AP Left } \\
\text { Opening }\end{array}$ & $\begin{array}{c}\text { AP Right } \\
\text { Opening }\end{array}$ & $\begin{array}{c}\text { AP Left } \\
\text { Closing }\end{array}$ & $\begin{array}{c}\text { AP Right } \\
\text { Closing }\end{array}$ & $\begin{array}{c}\text { AP Left } \\
\text { Opening }\end{array}$ & $\begin{array}{c}\text { AP Left } \\
\text { Closing }\end{array}$ & $\begin{array}{c}\text { AP Right } \\
\text { Opening }\end{array}$ & $\begin{array}{c}\text { AP Right } \\
\text { Closing }\end{array}$ \\
\hline Average & 3.71 & 2.42 & 2.71 & 1.97 & 3.71 & 2.71 & 2.42 & 1.97 \\
\hline Standard Deviation & 7.73 & 5.19 & 3.49 & 2.69 & 7.73 & 3.49 & 5.19 & 2.69 \\
\hline " $p$ " value & \multicolumn{2}{|c|}{0.01} & \multicolumn{2}{|c|}{0.001} & & & 0.3 & \\
\hline
\end{tabular}

(AP Left Opening - Amplitude peak in left joint during jaw opening; AP Right Opening - Amplitude peak in right joint during jaw closing; AP Left Closing

- Amplitude peak in left joint during jaw closing; AP Right Closing - Amplitude peak in right joint during jaw closing).

the movements of opening and closing on two joints (Figures 6 and 7). On analyzing joint sounds in the control group, there was no statistical difference between the left and right joints, and between the opening and closing movements (Table 2).

Moreover, during the opening movement between left and right TMJ, the left TMJ is presented with the amount of vibration energy (peak frequency - Hertz) higher to the opposite TMJ (Table 3 and Figure 8); while in closing the jaw, peak frequency was higher on the left (Table 3 and Figure 9).

Between the opening and closing movements of the left TM] there was a significant difference, with higher frequency peak on opening movement in relation to the jaw closing, as shown in (Table 3 and Figure 10). For right TMJ, there was a higher peak frequency during jaw closing (Table 3 and Figure 11). In the control group the difference in peak frequency between the right and left TMJ was not found. As well no difference was found between the opening and closing in jaw movement (Table 4).

The investigation of the relationship between sounds and joint severity of TMD was performed using the statistical test "Spearman Correlation", which compares the correlation values at each joint with the values found in the CMI. There was a significant correlation between the CMI and the frequency peak in the opening left (Table 6 and Figure 12). There was no significant correlation between the CMI and the amplitude peak in left TMJ on opening, amplitude peak in right TMJ on opening, amplitude peak in left TMJ on closing and amplitude peak in right TMJ on closing (Table 5). In the same way, there was no significant correlation between the CMI and the peak frequency in right TMJ on opening, frequency peak in left TMJ on closing and frequency peak in right TMJ on closing (Table 6).

\section{Discussion}

The high prevalence of females found in the sample studied $(87.5 \%)$ is similar to earlier studies in the literature $[10,11]$; also the age range 30.83 , is comparable to that described in other studies [12,13]. Differences in the clinical protocols used to establish TMD diagnoses may be responsible for the high variability of results between studies reported by past reviews [14]. In this study we used the RDC/TMD, introduced in 1992 by Dworkin and Leresche, with the aim of obtaining a standardized diagnosis of intra-articular TMD most commonly found, enabling greater reliability to clinical research $[7,14,15]$. The RDC/TMD provide criteria for a dual-axis diagnosis, the patient receives a physical diagnosis (axis I) along with a psychosocial assessment (axis II). According to axis I, in this protocol the subjects were classified into 3 groups: group I (myofascial pain), group II (disc displacement) and group III (arthralgia, osteoarthritis, osteoarthritis). Regarding the results found in the RDC, a study of systematic literature evaluating 3,463 subjects found a higher prevalence of abnormal muscle (group I) and changes in the positioning of the articular disc (group II), $45.5 \%$ and $41.1 \%$, respectively [16].

During clinical practice and research, it is important to assess the severity of signs and symptoms; therefore, the Craniomandibular Index (CMI) was developed, in order to provide a standardized measurement of the severity of the changes related to TMJ, including problems in jaw movement, joint sounds, joint sensibility and muscle [8]. The CMI consists of 62 items that are coded as positive or negative. In addition 


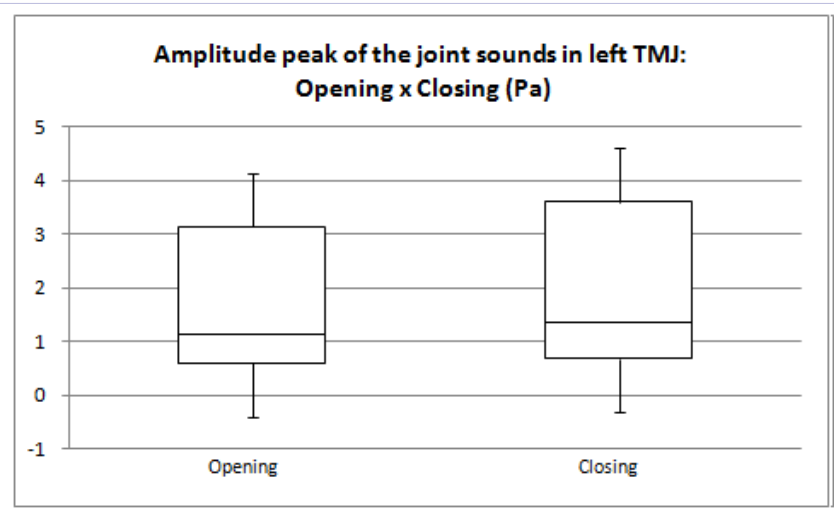

Figure 6: Mean and standard deviation of the amplitude peak of joint sounds in the left TMJ, during opening and closing movement.

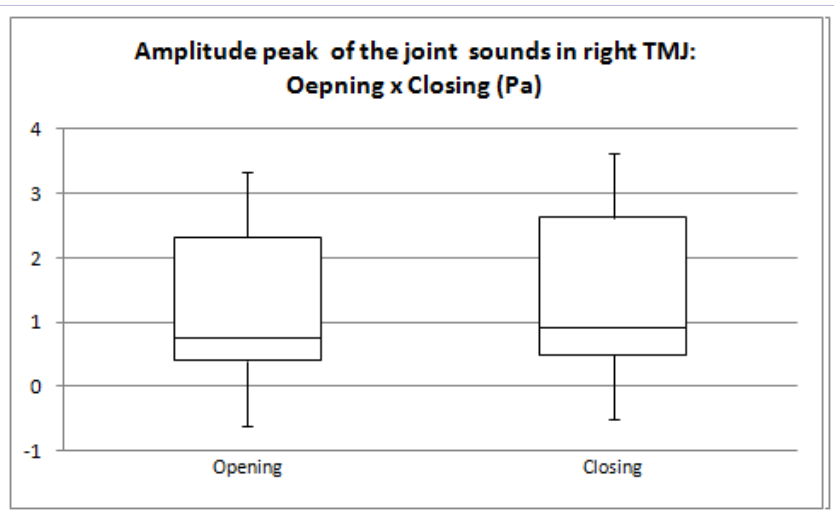

Figure 7: Mean and standard deviation of the amplitude peak of joint sounds in the right TMJ, during opening and closing movement.

Table 2: Amplitude peak of joint sounds in subjects with no temporomandibular disorders sings and symptoms. (AP Left Opening - Amplitude peak in left joint during jaw opening; AP Right Opening - Amplitude peak in right joint during jaw closing; AP Left Closing - Amplitude peak in left joint during jaw closing; AP RightClosing - Amplitude peak in right joint during jaw closing).

\begin{tabular}{|l|c|c|c|c|c|c|c|c|}
\hline & $\begin{array}{c}\text { AP Left } \\
\text { Opening }\end{array}$ & $\begin{array}{c}\text { AP Right } \\
\text { Opening }\end{array}$ & $\begin{array}{c}\text { AP Left } \\
\text { Closing }\end{array}$ & $\begin{array}{c}\text { AP Right } \\
\text { Closing }\end{array}$ & $\begin{array}{c}\text { AP Left } \\
\text { Opening }\end{array}$ & $\begin{array}{c}\text { AP Left } \\
\text { Closing }\end{array}$ & $\begin{array}{c}\text { AP Right } \\
\text { Opening }\end{array}$ & $\begin{array}{c}\text { AP Right } \\
\text { Closing }\end{array}$ \\
\hline Average & 0.65 & 0.38 & 1.06 & 0.86 & 0.65 & 1.06 & 0.38 & 0.86 \\
\hline Standard Deviation & 0.898 & 0.51 & 0.92 & 1.197 & 0.898 & 0.92 & 0.51 & 1.197 \\
\hline " $p$ " value & \multicolumn{2}{|c|}{0.57} & \multicolumn{2}{|c|}{0.17} & & 0.12 \\
\hline
\end{tabular}

to an overall aggregate score (CMI); the CMI produces a joint Dysfunction Index (DI) and a Muscle Index (MI). The DI quantifies jaw range and motion; TMJ sounds such as clicking, popping, and crepitus. Wherein, the MI quantifies pain in the muscles of the neck, face and palpitations in the shoulder muscles [17]; CMI values range from 0 to 1 , with the average value determined as $0.519 \pm 0.175$ (found by the sum of the subdivisions of the CMI (DI and MI) divided by 2). Similar results were found in a study which evaluated 19 patients with TMD in two days, on the first day the mean and standard deviation found in the CMI was $0.39 \pm$ 0.12 and on the second day, it was $12.41 \pm 00.13$ [8].

Joint sounds may be present in asymptomatic joints [4]; however, the presence of noise joint with or without pain may be indicative of the presence of TMD and may indicate changes in the lubrification of the joint, movement of the joint structures and changes in the anatomical shape. Computerized ultrasound equipment providing a standard differentiation between the various joint sounds; has been used to assist in the analysis of noise. Recording the noise helps in its classification and possible assessment of severity of changes in noise level; this allows analysis of noise in terms of intensity, frequency, duration and location during jaw opening and closing [9].

The analysis in the present study was focused on EVG parameters, peak amplitude and peak frequency, as these parameters were important in the evaluation of the data in relation to joint sounds, and also these were not extensively discussed in the literature.

The amplitude peak is the point of highest intensity of vibration in the joint, measured in Pascals $(\mathrm{Pa})$. The values in asymptomatic patients, 0.38 to 1.06 (Table 2), were comparable to those found in the literature, 0.22 to $0.51 \mathrm{~Pa}$ [18] reaching up to $1 \mathrm{~Pa}$ [19]. In patients with TMD studies the values were up to 8.4 Pa; while in patients with only inflammatory TMD, the values were less pronounced, approximately 0.35 to $3.96 \mathrm{~Pa}$. These values are similar to those found in the present study (Table 1).

Another important aspect to be analyzed in electrovibratography is in relation to the point where there is a greater amount of vibrational energy, called peak frequency and is measured in Hertz (Hz). In patients without signs and symptoms of TMD, the numerical values of the average peak frequency can be found between $37.5 \mathrm{~Hz}$ [19], similar results are found in this study (Table 4), but another study shows $71 \mathrm{~Hz}$ values during the opening cycle and $59.5 \mathrm{~Hz}$ during the closing [20]. One study evaluated the peak frequency in patients with

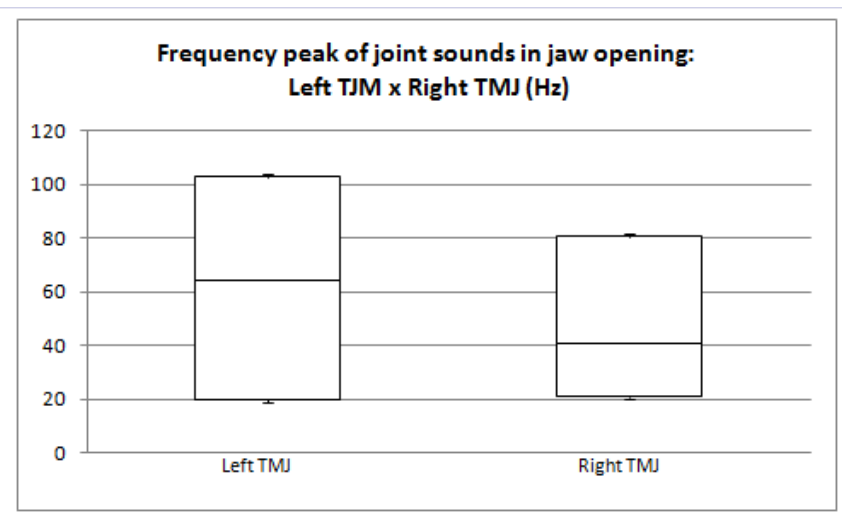

Figure 8: Mean and standard deviation of the frequency peak of the joint sounds during jaw opening, TMJ left and right TMJ. 
Table 3: Frequency peak of joint sounds in subjects with temporomandibular disorders. (FP Left Opening - Frequency peak in left joint during jaw opening; FP Right Opening - Frequency peak in right joint during jaw closing; FP Left Closing - Frequency peak in left joint during jaw closing; FP Right Closing - Frequency peak in right joint during jaw closing).

\begin{tabular}{|l|c|c|c|c|c|c|c|c|}
\hline & $\begin{array}{c}\text { FP Left } \\
\text { Opening }\end{array}$ & $\begin{array}{c}\text { FP Right } \\
\text { Opening }\end{array}$ & $\begin{array}{c}\text { FP Left } \\
\text { Closing }\end{array}$ & $\begin{array}{c}\text { FP Right } \\
\text { Closing }\end{array}$ & $\begin{array}{c}\text { FP Left } \\
\text { Opening }\end{array}$ & $\begin{array}{c}\text { FP Left } \\
\text { Closing }\end{array}$ & $\begin{array}{c}\text { FP Right } \\
\text { Opening }\end{array}$ & $\begin{array}{c}\text { FP Right } \\
\text { Closing }\end{array}$ \\
\hline Average & 71.31 & 52.09 & 47.99 & 39.14 & 71.31 & 47.99 & 52.09 & 71.31 \\
\hline Standard Deviation & 70.41 & 45.35 & 52.46 & 38.02 & 70.41 & 52.46 & 45.35 & 38.02 \\
\hline " $p$ " value & \multicolumn{2}{|c|}{0.01} & \multicolumn{2}{|c|}{0.02} & & 0.02 \\
\hline
\end{tabular}

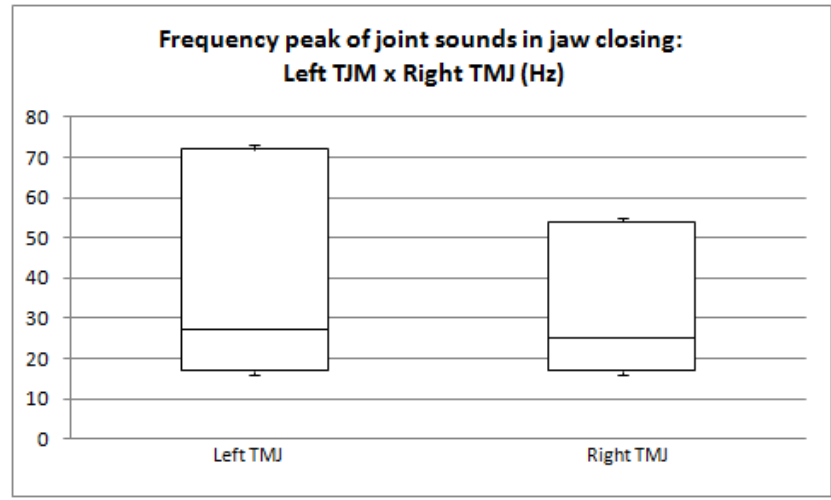

Figure 9: Mean and standard deviation of the frequency peak of the joint sounds during jaw closing, TMJ left and right TMJ.

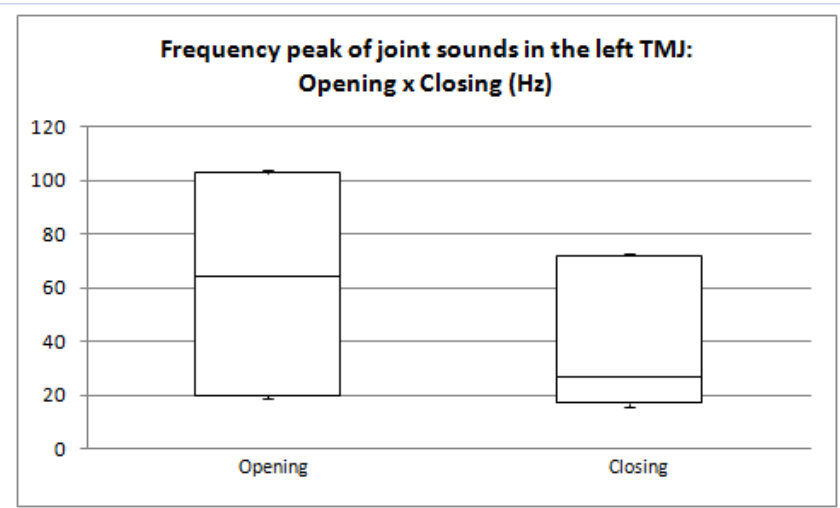

Figure 10: Mean and standard deviation of the frequency peak of joint sounds in the left TMJ, during opening and closing movement.

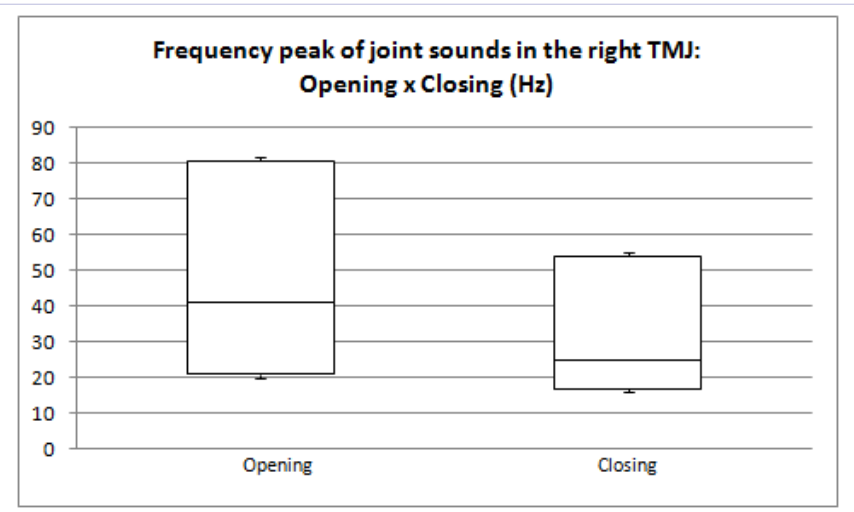

Figure 11: Mean and standard deviation of the frequency peak of joint sounds in the right TMJ, during opening and closing movement.

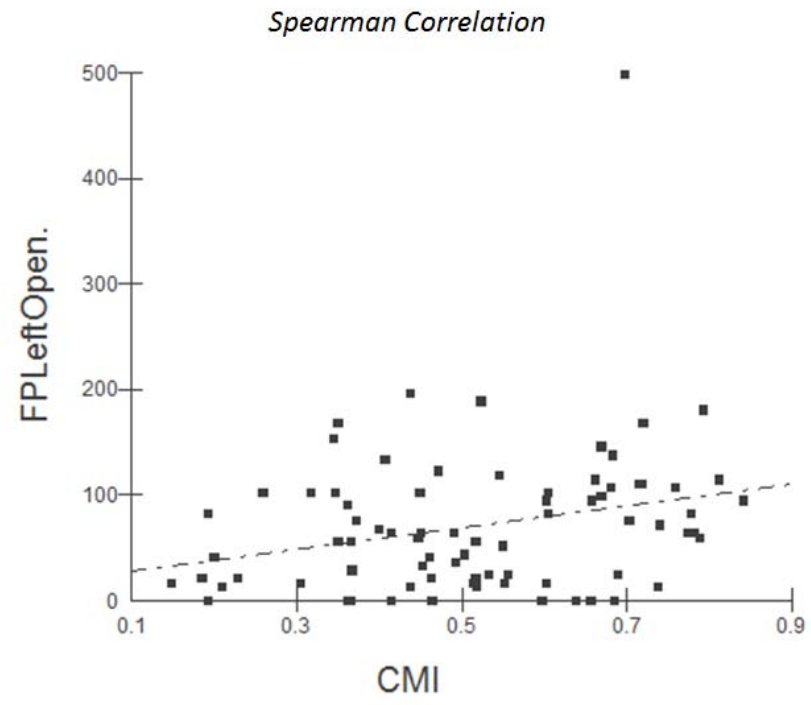

Figure 12: Correlation between the frequency peak of joint sounds on opening left TMJ with TMD severity determined by the Craniomandibular Index (CMI) - p = 0.02 (Spearman's Correlation).

disc displacement with reduction and disk displacement without reduction, finding values close to those found in this study (Table 3 ), and in patients with disc displacement with reduction, during the opening movement, the peak frequency was around $64.2 \mathrm{~Hz}$ and $58.6 \mathrm{~Hz}$ closing. Already in patients with disc displacement without reduction values occurred around $55.5 \mathrm{~Hz}$ to $31.0 \mathrm{~Hz}$ in jaw opening and closing [21]. However, peak values of frequency of TMD were also found around $131.3 \mathrm{~Hz}$ during the opening movement of the jaw and $68.5 \mathrm{~Hz}$ during closing [20]. In patients with joint inflammation, these values were found around 83.2 to $120.2 \mathrm{~Hz}$, where the greatest amount of vibration energy was found in the middle of the opening cycle [18].

The analysis of data reveals the presence of outliers, probably due to non- homogenous study sample using subjects selected from a public school, and also due to the fact that the differences between gender and age were not taken into consideration during the selection of subjects. In addition, the severity was assessed only by CMI tests; articular pathologies were not used to verify additional images, during the study.

It is important to examine the peak amplitude and peak frequency, as healthy joints present sounds at low frequency; however, as a result of pathological changes, the noise amplitude decreases and the frequency increases in all the phases of jaw 
Table 4: Frequency peak of joint sounds in subjects with no temporomandibular disorders signs and symptoms. (FP Left Opening - Frequency peak in left joint during jaw opening; FP Right Opening - Frequency peak in right joint during jaw closing; FP Left Closing - Frequency peak in left joint during jaw closing; FP Right Closing - Frequency peak in right joint during jaw closing).

\begin{tabular}{|l|c|c|c|c|c|c|c|c|}
\hline & $\begin{array}{c}\text { FP Left } \\
\text { Opening }\end{array}$ & $\begin{array}{c}\text { FP Right } \\
\text { Opening }\end{array}$ & $\begin{array}{c}\text { FP Left } \\
\text { Closing }\end{array}$ & $\begin{array}{c}\text { FP Right } \\
\text { Closing }\end{array}$ & $\begin{array}{c}\text { FP Left } \\
\text { Opening }\end{array}$ & $\begin{array}{c}\text { FP Left } \\
\text { Closing }\end{array}$ & $\begin{array}{c}\text { FP Right } \\
\text { Opening }\end{array}$ & $\begin{array}{c}\text { FP Right } \\
\text { Closing }\end{array}$ \\
\hline Average & 39 & 37 & 25 & 25 & 39 & 25 & 37 & 25 \\
\hline Standard Deviation & 52.04 & 41.03 & 17.02 & 19.18 & 52.04 & 17.02 & 41.03 & 17.02 \\
\hline "p" value & \multicolumn{2}{|c|}{0.87} & \multicolumn{2}{|c|}{0.94} & & 0.30 \\
\hline
\end{tabular}

Table 5: Spearman's correlation with Craniomandibular Index and Amplitude Peak of joint sounds.

\begin{tabular}{|c|c|c|c|c|}
\hline & $\begin{array}{c}\text { AP Left } \\
\text { Opening }\end{array}$ & $\begin{array}{c}\text { AP Right } \\
\text { Opening }\end{array}$ & $\begin{array}{c}\text { AP Left } \\
\text { Closing }\end{array}$ & $\begin{array}{c}\text { AP Right } \\
\text { Closing }\end{array}$ \\
\hline Spearman correlation coefficient & 0.006 & 0.002 & 0.006 & 0.07 \\
\hline " $p$ " value & 0.95 & 0.98 & 0.95 & 0.51 \\
\hline
\end{tabular}

Table 6: Spearman's correlation with Craniomandibular Index and Frequency Peak of joint sounds.

\begin{tabular}{|c|c|c|c|c|}
\hline & $\begin{array}{c}\text { FP Left } \\
\text { Opening }\end{array}$ & $\begin{array}{c}\text { FP Right } \\
\text { Opening }\end{array}$ & $\begin{array}{c}\text { FP Left } \\
\text { Closing Right } \\
\text { Closing }\end{array}$ \\
\hline Spearman correlation coefficient & 0.25 & 0.05 & 0.09 & 0.08 \\
\hline " $p$ "value & 0.02 & 0.64 & 0.39 & 0.45 \\
\hline
\end{tabular}

movement $[22,23]$. Higher vibration energy (frequency peak) of sound predicts deterioration in the patient's condition.

The sounds with very high amount of energy are considered as the crepitus type sounds; while the sounds with less energy (lower frequency), but a high amplitude are featured as loud sounds, as found in clicks [22]. In this study it was confirmed that noise with high amplitude peak is not always related to the severity of the dysfunction. Because not all patients, in whom the clicks sound was recorded, complain of pain or any other sign of TMD [4]. On the other hand low values of amplitude peak (Pa) in subjects with intra-articular TMD could be associated with inflammatory diseases [18]. The severity of TMD is more related to inflammation than the intensity $(\mathrm{Pa})$ of joint sounds (the presence of clicks), because in a lot of cases joint sounds happen without the presence of pain.

However, when the noise has high vibration energy (frequency peak), with low amplitude peak, there is no association with the severity of TMD; but in few circumstances where the peak is characteristic of very high frequency crepitus, it is associated with degenerative diseases [23]. This might explain the positive correlation between CMI and frequency peak during the opening movement in left TMJ.

The data found in the literature determined that the electrovibratography is for understanding and formulating hypotheses about changes in intra-articular TMJ and perhaps muscular changes that influence them, as the large number of subjects had muscle dysfunction. The correlation between the frequency peaks in the left TMJ, during opening, suggests that the EVG can assist in evaluating the severity of joint dysfunction, enriching clinical findings.

\section{Conclusion}

The electrovibratography has contributed to the understanding of the severity of intra-articular dysfunction and their relationship with the joint sounds. However, from the data found in the study, it is possible to emphasize that electrovibratography analysis should be performed in conjunction with clinical evaluation, because when observed separately, the relationship between the noise type and severity is not always well established.

\section{Acknowledgements}

The authors are grateful for the donation of the electrovibratography device made by FAPESP and financial support throughout the research granted by CAPES.

This study was presented during the 30th Annual Meeting of the Society for Dental Research (IADR Brazilian Division), which occurred in the period of 05 to 08, September 2013.

\section{References}

1. Cooper BC, Kleinberg I. Examination of a large patient population for the presence of symptoms and sings of temporomandibular disorders. Cranio. 2007; 25(2):114-26.

2. De Leeuw R, Klasser GD. Orofacial Pain: Guidelines for Assessment, Diagnosis, and Management. 5th edn. Illinois: Quintessence Publishing Co Inc, 2013.

3. Turk DC. Psychosocial and behavioral assessment of patients with temporomandibular disorders: diagnostic and treatment implications. Oral Surg Oral Med Oral Pathol Oral Radiol Endod. 1997; 83(1):65-71.

4. Tanzilli RA, Tallents RH, Katzberg RW, Kyrkanides S, Moss ME. Temporomandibular joint sound evaluation with an electronic device and clinical evaluation. Clin Orthod Res. 2001; 4(2):72-78.

5. Radke JC, Kull RS. Distribution of Temporomandibular Joint Vibration Transfer to the Opposite Side. Cranio. 2012; 30(3):194-200. 
6. Mazzetto MO, Hotta TH, Carrasco TG, Mazzetto RG. Characteristics of TMD Noise Analyzed by Electrovibratography. Cranio. 2008, 26(3):222-8

7. Dworkin SF, Leresche L. Research diagnostic criteria for temporomandibular disorders: review, criteria, examinations and specifications, critique. J Craniomandib Disord. 1992; 6(4): 301-55.

8. Fricton JR, Schiffman EL. Reliability of a Craniomandibular Index. Journal Dental Research.1986; 65(11): 1359-1364

9. Mazzetto MO, Hotta TH, Mazzetto RG. Analysis of TMJ vibration sounds before and after use two types of occlusal splints. Braz Dent J. 2009; 20(4):325-30.

10. Nomura K, Vitti M, Oliveira AS, Chaves TC, Semprini M, Siéssere S, et al. Use of the Fonseca's Questionnaire to Assess the Prevalence and Severity of Temporomandibular Disorders in Brazilian Dental Undergraduates. Braz Dent J. 2007; 18(2):163-7.

11. Katsoulis K, Bassetti R, Getaz IW, Stern MR, Katsoulis J Temporomandibular disorders/myoarthropathy of the masticatory system. Schweiz Monatsschr Zahnmed. 2012; 122(6): 510-518.

12. Leader JK, Boston JR, Rudy TE, Greco CM. Zaki HS. The influence of mandibular movements on joint sounds in patients with temporomandibular disorders. J Prosthet Dent. 1999; 81(2): 186-95.

13. Calderon PS, Hilgenberg PB, Rossetti LMN, Laurenti JVH, Conti PCR. Influence of tinnitus on pain severity and quality of life in patients with temporomandibular disorders. J Appl Oral Sci. 2012; 20(2): 170173.

14. Manfredini D, Guarda-Nardini L, Winocur E, Piccotti F, Ahlberg J, Lobbezzoo F. Research diagnostic criteria for temporomandibular disorders: a systematic review of axis I epidemiologic findings. Oral
Surg Oral Med Oral Pathol Oral Radiol Endod. 2011; 112 (4): 453-462.

15. Hasanain F, Durham J, Moufti A, Steen ON, Wassell RW. Adapting the diagnostic definitions of the RDC/TMD to routine clinical practice: A feasibility study. J Dent. 2009; 37(12): 955-62. doi: 10.1016/j. jdent.2009.08.001.

16. Kim YK, Kim SG, Im JH, Yun PY. Clinical surveu of the patients with temporomandibular joint disorders, using Research Diagnostic Criteria (Axix II) for TMD: Preliminary study. J Craniomaxillofac Surg. 2012; 40(4): 366-72. doi: 10.1016/j.jcms.2011.05.018

17. Hatch JP, Rugh JD, Sakai S, Prihoda TJ. Reliability of the Craniomandibular Index. J Orofac Pain. 2002; 16(4):284-95.

18. Garcia AR, Madeira MC, Paiva G, Olivieri KAN. Joint Vibration Analysis in Patients with Articular Inflammation. Cranio. 2000; 18(4):272-9.

19. Christensen LV, Orloff J. Reproducibility of temporomandibular joint vibrations (electrovibratography). J Oral Rehabil. 1992; 19(3): 253 263.

20. Toolson GA, Sadowsky C. An evaluation of the relationship between temporomandibular joint sounds and mandibular movements. J Craniomandib Disord Facial Oral Pain 1991; 5(3): 187-196

21. Tallents RH, Hatala M. Temporomandibular joint sounds in asymptomatic volunteers. J Prosthet Dent 1993; 69(3): 298-304

22. Hutta JL, Morris TW, Tallents RH. Separation of internal derangements of the temporomandibular joint using sound analysis. Oral Surg Oral Med Oral Pathol. 1987; 63(2): 151-157

23. Olivieri KAN, Garcia AR, Paiva G, Stevens C. Joint Vibrations Analysis in Asymptomatic Volunteers and Symptomatic Patients. Cranio. 1999 Jul;17(3):176-83. 